\title{
Malaria outbreak investigation in Mecha, Dera and Fogera districts, Amhara region, Ethiopia
}

\author{
Mulugojjam Andualem Tamiru², Addisu Workineh Kassa ${ }^{1,3}$, Belay Bezabih Beyene ${ }^{1,3}$, \\ Tilahun Belete Mossie ${ }^{4}$, Yeshiwork Amogne Mekonnen ${ }^{3}$ \\ ${ }^{1}$ Public Health Emergency Management, Bahir-Dar, Ethiopia \\ ${ }^{2}$ Bahir-Dar University, Bahir-Dar, Ethiopia \\ ${ }^{3}$ Amhara Regional Health Bureau, Bahir-Dar, Ethiopia \\ ${ }^{4}$ Felegehiwot Referral Hospital, Bahir-Dar, Ethiopia
}

\section{Email address:}

anamek9800@gmail.com (M. A. Tamiru), analid9800@gmail.com (A. W. Kassa)

\section{To cite this article:}

Mulugojjam Andualem Tamiru, Addisu Workineh Kassa, Belay Bezabih Beyene, Tilahun Belete Mossie, Yeshiwork Amogne Mekonnen. Malaria Outbreak Investigation in Mecha, Dera and Fogera Districts, Amhara Region, Ethiopia. American Journal of Health Research. Vol. 2, No. 4, 2014, pp. 182-187. doi: 10.11648/j.ajhr.20140204.23

\begin{abstract}
Introduction- Malaria is caused by the protozoan parasite plasmodium and transmitted by anopheles mosquitoes. It remains the major public health challenge in Amhara region. Mecha, Dera and Fogera are some of the malaria endemic districts of the region. The aim of this study was to investigate the outbreak and guide intervention measures. Methods Descriptive cross sectional investigation of malaria outbreak was conducted. We used health facility records of malaria data and entomological survey. We discussed with health extension workers and available morbidity, mortality and diagnostic data was collected. We surveyed households for clinical malaria cases and utilization of LLINs and its status, the condition of IRS operation at household level was observed. Results-In Midre-Genet kebele/village the prevalence rate of malaria in the $4^{\text {th }}$ week of April was 3 per 1000(19) population and reached to 37 per 1000(226) population in the $2^{\text {nd }}$ week of May 2012.The attack rate was 82.5 per 1000(67) population in under-fives and 82.6 per 1000(429) population in $\geq 5$ years old. In Wotet-Ber kebele the prevalence rate of malaria increased to 5 per 1000(35) population in the $1^{\text {st }}$ week of May 2012 and became 22 per 1000 (150) population in the $2^{\text {nd }}$ week of May. The attack rate was 30.4 per 1000(28) population in under-fives and 42.9 per 1000 (253) population in $\geq 5$ years old. In Zemene-Hiwot kebele/village the prevalence rate of malaria increased to 4.5 per 1000 (45) population in the $1^{\text {st }}$ week of May 2012 and became 17 per 1000(171) population in the $3^{\text {rd }}$ week of May 2012 . The attack rate was 82.6 per 1000(111) population in under-fives and 36.3 per 1000(312) in $\geq 5$ years old.In Hamusit cluster the number of confirmed malaria cases surpass the threshold starting from December 2011. Attack rate was 8.9 per 1000(559) population in December 2011 and became 14.7 per 1000(919) population in April 2012. In Aba Kiros kebele prevalence rate of malaria increased in the $2^{\text {nd }}$ week of May $20121.2(8)$ and reached to 14.9(93) per 1000 population in the $1^{\text {st }}$ week of June 2012. Vector control interventions were not done in all affected villages/kebeles. Conclusion- There were multiple breeding sites where the larvae of anopheles mosquitoes found and vector control interventions were not carried out timely. There was no weekly monitoring chart at districts and health facilities to detect increased malaria cases at an early stage.
\end{abstract}

Keywords: Plasmodium, Malaria, Mecha, Fogera, Dera, Ethiopia

\section{Introduction}

Malaria remains one of the main global health problems of our time, causing more than one million deaths per year, with about $90 \%$ of deaths and $60 \%$ of cases occurring in Africa south of the Sahara. It is caused by the protozoan parasite plasmodium and transmitted by anopheles mosquitoes, which bite mainly between sunset and sunrise[1].

In Ethiopia malaria is mainly seasonal with unstable transmission in the highland fringe areas and of relatively longer transmission duration in lowland areas, river basins and valleys. Anopheles arabiensis is the main malaria vector; 
anopheles pharoensis, anopheles funestus and anopheles nili play a role as secondary vectors[2], [3] [4].

Improved epidemic preparedness, greatly reduce the risk of recurrent epidemics[3],[5]. Indoor residual spraying (IRS) in Ethiopia has been one of the most popular interventions for malaria control. IRS requires high coverage if any protection is to be achieved, and the required coverage must be achieved before and throughout the transmission season. Timeliness is particularly important in the control of outbreaks. Effective coverage should be maintained during the entire transmission season implies that spraying of the whole area to be protected should be completed before the beginning of that season[6], [7], [8].

Malaria remains to be the major public health challenge in Amhara region. Mecha, Dera and Fogera districts are some of the malaria endemic districts of the region.

Investigation was conducted in collaboration with district health offices and zonal health departments to confirm and verify outbreaks of malaria and implementing control and preventive measures [3], [9], [10], [11].

The aim of investigation was to investigate the occurrence of malaria outbreak, identify the risk factors and suggest practical control measures to alleviate the disease burden of the community in Mecha, Dera and Fogera districts, West Gojjam and South Gondar zones,

\section{Methodology}

\subsection{Study Areas}

Mecha, Dera and Fogera districts are some of the malaria endemic districts of the region. Mecha district covers $15602 \mathrm{sq} . \mathrm{km}$ and has 40 rural \& four urban kebeles/village. Rainfall ranges from $1500-2000 \mathrm{~mm}$ per annum; average temperature ranges $20-260 \mathrm{c}$. The district is found $37.13^{\circ} \mathrm{E}$ longitude and $11.35^{\circ} \mathrm{N}$ latitude. Dera is found at $37.6^{\circ} \mathrm{E}$ longitude and $11.7^{\circ} \mathrm{N}$ latitude and Fogera is found $37.6^{\circ} \mathrm{E}$ longitude $11.86^{\circ} \mathrm{N}$ latitude.

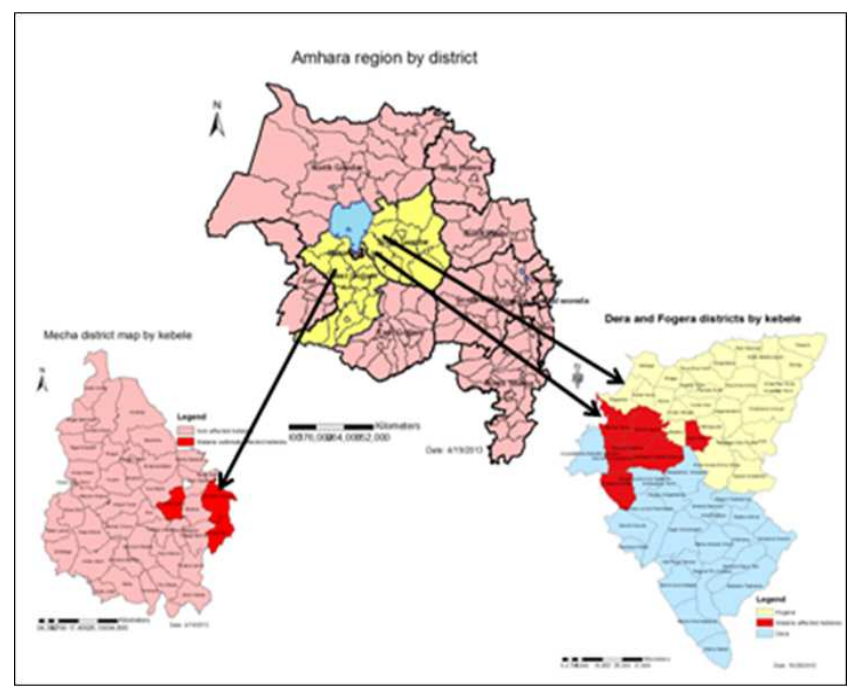

Figure 1. Outbreak affected kebeles in Mecha, Dera and Fogera districts

\subsection{Study Design}

Descriptive cross sectional study was conducted in all villages (localities).

\subsubsection{Observation}

Utilization of Long Lasting Impregnated Nets (LLINs) and its status, the condition of Indore Residual Spraying (IRS) operation at household level was observed. Mosquito breeding sites were also visited in the villages (localities).

\subsection{Study Period}

Study was undertaken in 15-23 May 2012 in Mecha and 2-5 June 2012 in Dera and Fogera districts.

\subsection{Data Collection Method}

We used retrospective health facility records of malaria data from daily registers which consist of age, sex, residence (village or got), date of onset, date seen at health facility and species collected from the health post. We discussed with health extension workers and available morbidity, mortality and diagnostic data was collected. We surveyed households for clinical malaria cases and interviewed households using semi structured questionnaire in order to collect risk factors for the malaria outbreak.

We compiled daily data to weekly total cases in the affected localities in the previous weeks and months compared with the number of cases of the weeks and months of 2012.The available daily data then converted to week and compared with the same week of the threshold to define the rapid increase of malaria cases.

\subsection{Data Analysis}

Data was entered in a computer after transcribing from a hard copy which used to collect the raw data from registries. Then the data had been cleaned and analyzed using Micro Soft excel.

\section{Results}

\subsection{Mecha District}

There was only one Health Extension Worker (HEW) in Midre-Genet kebele health post, Wotet-Ber and Zemene-Hiwot health posts had two HEWs each. We reviewed 2,648 malaria cases from daily records of malaria $\log$ book; 654 from Midre-Genet from July 2011 to15 May 2012; $48.5 \%$ ( 317) were females and 829 from Wotet-Ber with one death $(\mathrm{CFR}=0.12 \%)$ with mixed infection of malaria which was referred to nearest health center on 16 May 2012; $49.7 \%$ ( 412) were females. And 1165 malaria cases were reviewed from Zemene-Hiwot health post from July 2010 to 23 May 2012; 46.7 \%( 544) were females.

In Midre-Genet kebele the prevalence rate of confirmed malaria cases in the $4^{\text {th }}$ week of April was 3 per $1000(19)$ population, and reached to 14 per 1000 (83) population in the $1^{\text {st }}$ week of May 2012 and increased to 37 per 1000(226) 
population in the $2^{\text {nd }}$ week of May 2012. The attack rate was 82.5 per $1000(67)$ population in under-fives and 82.6 per

1000 (429) population in $\geq 5$ years old (figure 4 ).

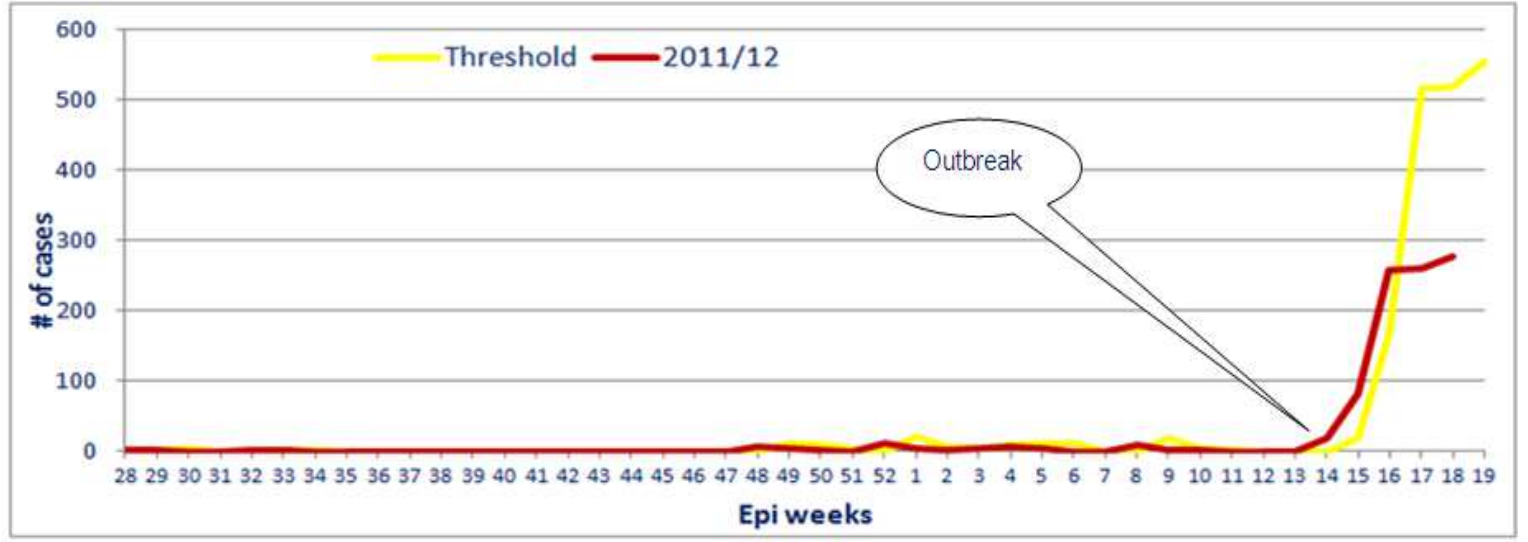

Figure 4. Malaria cases of Midre-Genet kebele from July 2011-May 2012

In Wotet-Ber kebele the prevalence rate of confirmed malaria cases increased to 5 per 1000(35) population in the $1^{\text {st }}$ week of May 2012 and 22 per 1000 (150) population in the $2^{\text {nd }}$ week of May 2012 as compared to the same weeks of the previous year (2011); 0.15(1) and 0.3(2) per 1000 population respectively. The attack rate was 30.4 per 1000 (28) population in under fives and 42.9 per $1000(253)$ population in $\geq 5$ years old (figure 5 ).

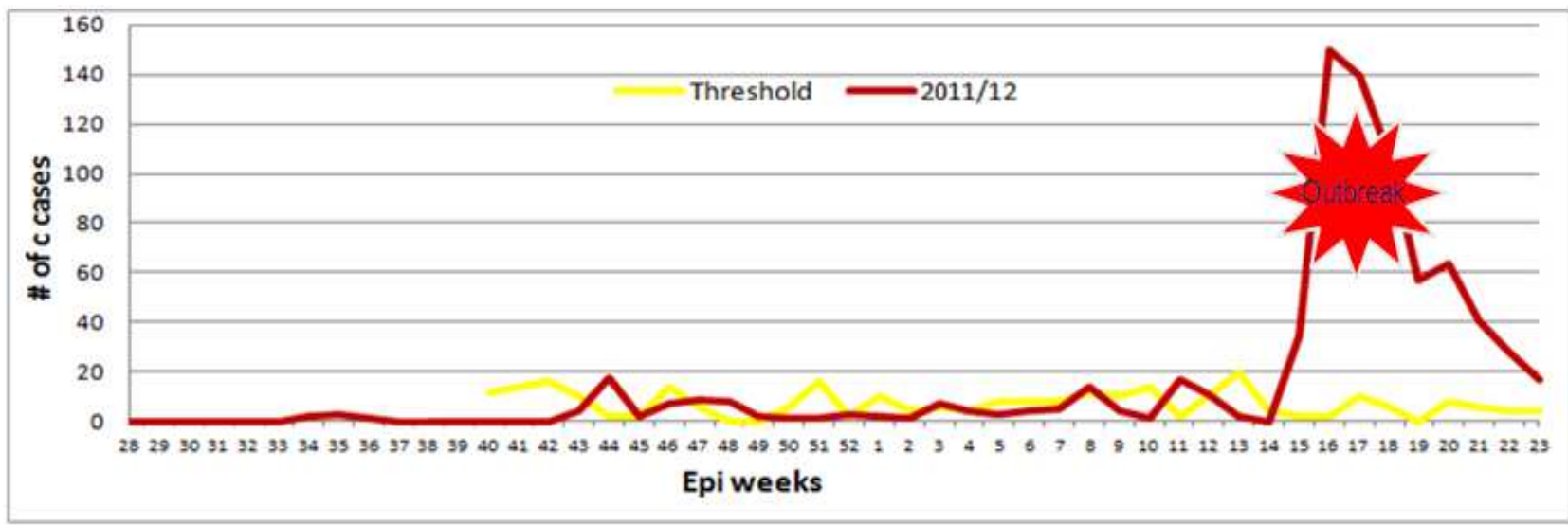

Figure 5. Confirmed malaria cases of Wotet-Ber kebele from July 2010-June 2012

In Zemene-Hiwot kebele the prevalence rate of confirmed malaria cases increased starting from $1^{\text {st }}$ week of May 2012; 4.5 per 1000 (45) population, 1.8 per $1000(18)$ population in the $2^{\text {nd }}$ week of May and 17 per 1000(171) population in the $3^{\text {rd }}$ week of May 2012 as compared to the weeks of the previous year (2011);0.4(4), 0.6(6) and 0.6(6) per 1000 populations respectively. The attack rate was 82.6 per $1000(111)$ population in under fives and 36.3 per $1000(312)$ in $\geq 5$ years old (figure 6 ).

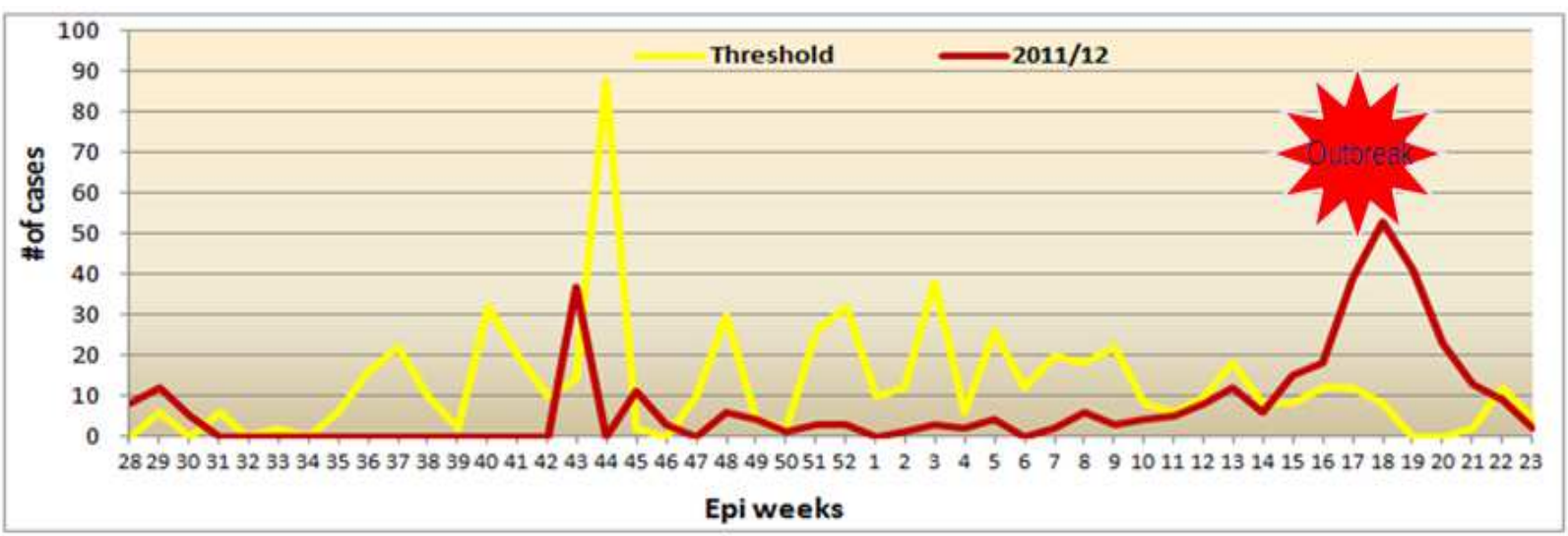

Figure 6. Confirmed malaria cases of Zemene-Hiwot kebele from July 2010-June 2012 


\subsection{Dera District}

We collected 6,836 malaria cases from daily records of malaria log book; 6,444 from Hamusit cluster health center from July 2011 to 5 June 2012. There was no death during the outbreak. Outbreak was started in December 2011 in Hamusit cluster.

In Hamusit cluster health center the number of confirmed malaria cases surpass the threshold starting from December
2011. $44 \%$ ( 897) of the cases were females. The attack rate was 8 per 1000(505) population in November 2011 and increased to 8.9 per 1000 (559) population in December 2011 and became 14.7per 1000 (919) population in April 2012. Attack rate was 8.9 per $1000(559)$ population in December 2011 and became 14.7 per 1000(919) population in April 2012(figure 7).

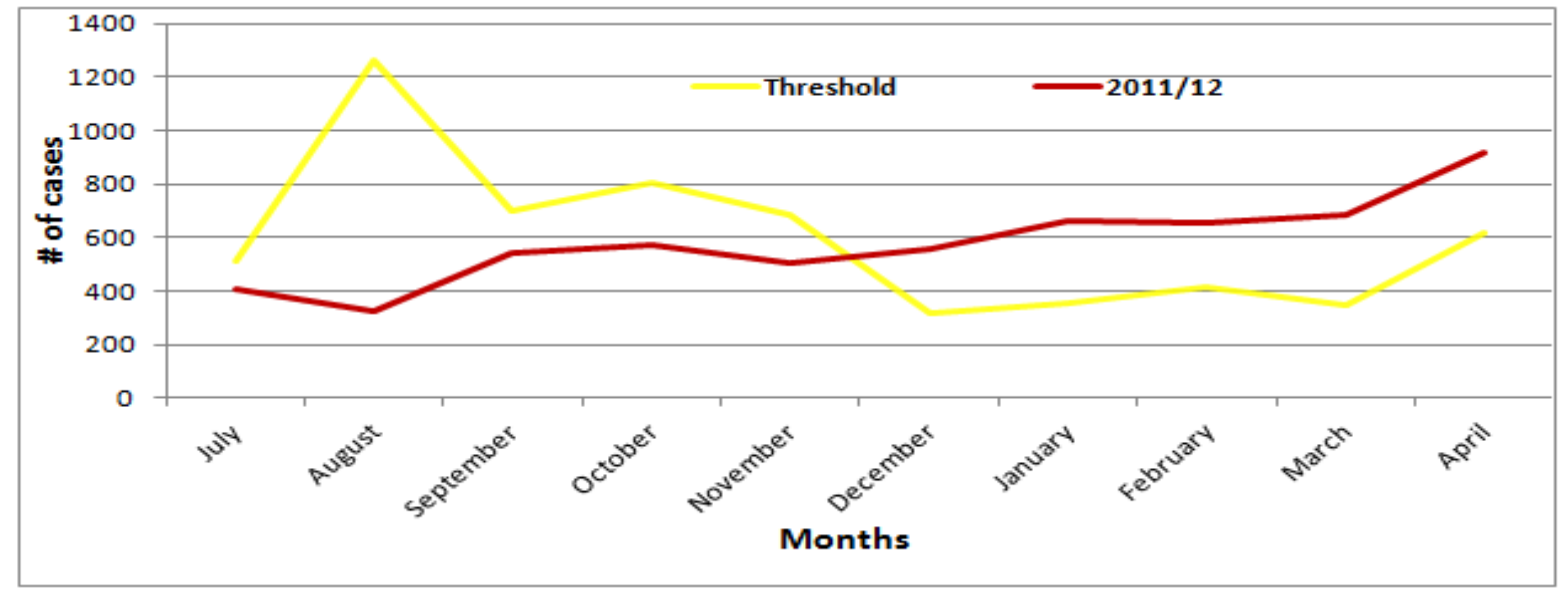

Figure 7. Confirmed malaria cases of Hamusit cluster health center from July 2011-April 2012

\subsection{Fogera District}

From Aba Kiros health post 392 cases were collected from August 2011 to 5 June 2012. Outbreak was started in the $2^{\text {nd }}$ week of May 2012 and there was no death.

Prevalence rate of confirmed malaria cases increased in the $2^{\text {nd }}$ week of May 2012 was 1.2(eight), in the last week of
May 4.8(30) and reached to 14.9(93) per 1000 population in the $1^{\text {st }}$ week of June as compared to the weeks in previous weeks; May $1^{\text {st }}$ week 0.3(2), May $3^{\text {rd }}$ week 0.6 (four) and May $4^{\text {th }}$ week was 4.8 per $1000(30)$ population. $48 \%$ ( 56) of the cases were females (figure 8).

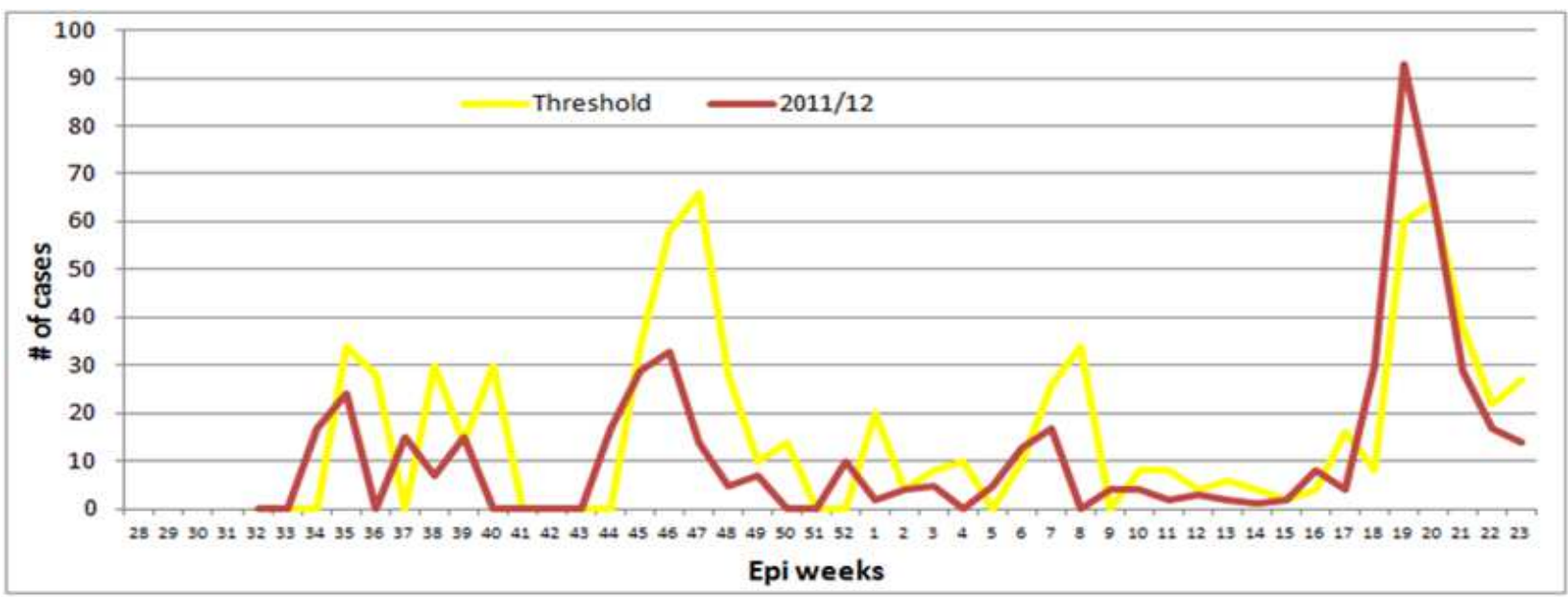

Figure 8. Confirmed malaria cases of Aba Kiros kebele, Fogera district from July 2011-June 2012.

\subsection{Observation}

Fifteen households from each of the affected localities were randomly visited to observe if malaria patients were found in households and the status of LLINs utilization. Most of the participants and interviewees did not use LLINs regularly. Only three of the fifteen in Midre-Genet, six of Wotet-Ber and five of Zemene-Hiwot kebeles respondents knew about the use of LLINs and used it regularly. Others perceived that it doesn't prevent malaria. With availability of LLINs, all study participants had at least one LLIN in their home. All visited households had LLINs hanged over the beds. However, nets in eighteen of forty five visited households had tears/holes.

Most of the participants of Aba Kiros kebele were not 
utilizing LLINs. Twelve of the fifteen respondents reported that they knew about the use of LLINs but only three of them using it. With availability of LLINs, all study participants had at least one LLIN in their home. Twelve of the respondents were used LLINs for different purposes such as making rope, as covering for grain and hay, curtain.

\section{Discussion}

The outbreak was caused by Anopheles mosquitoes localized in breeding places around the village and villagers in the districts. Outbreak affected population was 91,650 in three districts. The population was served by two health centers and three health posts in Mecha; two health centers and seven health posts in Dera and Fogera districts. It was an exaggerated seasonal increase precipitated by interruption of vector control activities. The districts known to be affected by outbreaks of malaria with high transmission all year round and become high from April to December and reached peaks in October. There were multiple breeding sites where the larvae of anopheles found. This might be due to a change in weather patterns for an unusual increase in the vector population, and interruption (breakage) of water bodies and irrigation (Nora-mender) and Gumara river in Fogera district were sources of the outbreak in affected areas can also lead to an increase in vector breeding sites because population who live around \& near the irrigation site was more affected. Moreover indoor residual spraying was not performed in affected localities of Dera and Fogera and IRS was not sprayed for many years in outbreak affected kebeles of Mecha district. Other vector control measures such as LLINs not replaced timely and abet chemical not sprayed for removing the larvae might result in seasonal peaks to levels that surpass the epidemic threshold and there was no intervention taken by the community in the year and HEWs did not work on environmental management (environmental manipulation or modification) in the districts.

Entomological survey was conducted in Midre-Genet kebele of Mecha and Aba Kiros kebele of Fogera districts where the majority of cases were coming first. There were multiple water bodies for breeding of Anopheles mosquitoes and they were not difficult to drain and fill but most of them never dry in short periods by themselves (figures 2 and 3 ).

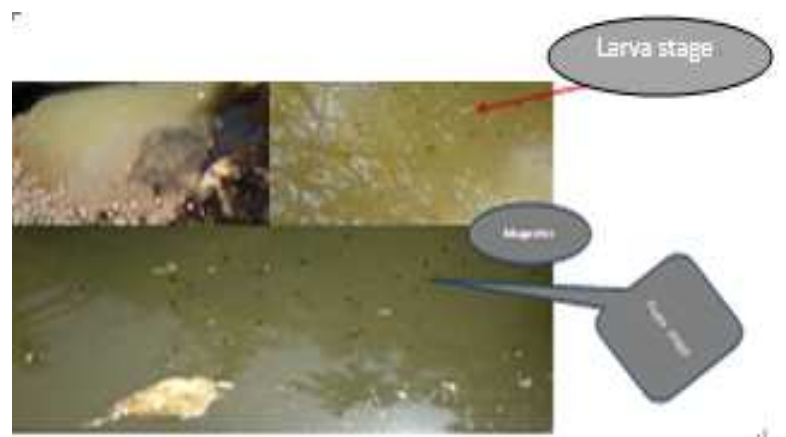

Figure 2. Some of the breading sites of outbreak affected kebeles of Mecha district

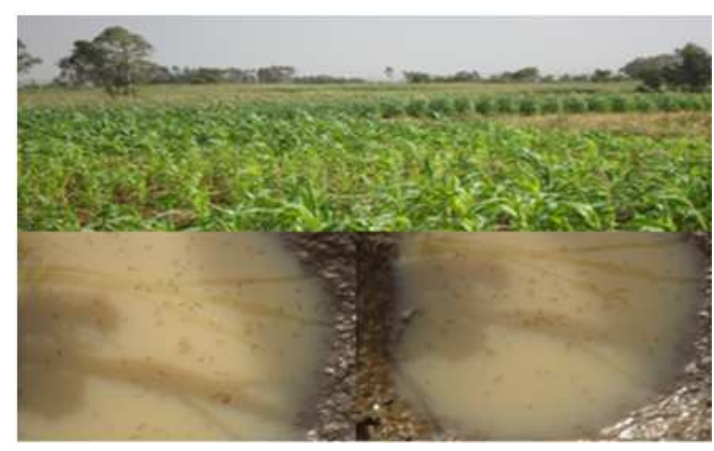

Figure 3. Some of the breading sites of Aba Kiros kebele of Fogera district

Malaria outbreak was recorded in areas where conditions for malaria transmission were unstable and the population lacks protective immunity; usually this is at the fringes of whichever climate factor limits malaria's distribution. In temperate climates and in tropical highlands, temperature restricts vector multiplication and the development of the parasite in the mosquito, while in arid climates precipitation restricts mosquito breeding. Epidemics in areas with tropical highland malaria have been reported from Ethiopia and Madagascar, in which above normal temperatures were suspected[6].

We used five years data by taking the $2^{\text {nd }}$ largest number as a threshold and comparing with 2012 for Hamusit cluster health center (figure 7).

And in Aba Kiros kebele since there was no complete five years or last year malaria data, we used doubling of cases starting from August 2011 in week basis. We identified the outbreaks of malaria and ongoing outbreak during assessment in the kebele (figure 8)

We used doubling of last year weekly(2010/11) malaria data for Wotet-Ber and Zemene-Hiwot kebeles but for the Midre-Genet kebele since there was no complete five years or last year malaria data we used doubling of cases starting from July 2011 in week basis. We identified the outbreaks of malaria and ongoing outbreak during assessment at each kebele. In all three kebeles of Mecha the number of malaria cases crossed the threshold starting from the $3^{\text {rd }}$ week of April 2012. During these weeks the number of cases who had mixed infection was more than double than cases that had mono species in Midre-Genet and Wotet-Ber kebeles but in Zemene-Hiwot kebele plasmodium falciparum was more than tripled than others and under-fives were more affected than adults. However, in all health posts had no weekly malaria epidemic monitoring chart for early detection of outbreaks.

In all kebeles that we saw the number of malaria cases crossed the threshold starting from December 2011 in Hamusit cluster health center and in the 1st week of May 2012 in Aba Kiros kebele. During these weeks the number of cases who had plasmodium falciparum infection was more than that of other cases. However, the health post and health center had no weekly malaria epidemic monitoring chart for early detection of outbreaks. 


\section{Conclusion}

All health facilities managing malaria cases using malaria diagnostic service based on national protocol.

Vector control interventions were not carried out timely in the districts.There was no district-based epidemic early warning, prevention and response team. The districts targeted for deltametrin spray but with shortage of operational budget (Dera and Fogera districts) outbreak affected localities were not sprayed. However logistic and personal protective equipments were given to the districts by the regional health bureau but no strong activities done to eradicate breeding sites for vectors with community participation. The collected data from facilities showed that outbreaks before they announced to the district health offices that crossed the threshold. The districts started response to the outbreak lagged by one week and actual interventions of LLINs replacement, spray with deltametrin and mobilization of the community started after two weeks of the outbreaks and after five months in Hamusit cluster.

There was no weekly monitoring chart or other systems to detect malaria outbreaks at district and health facility level. Health extension workers not reported regularly to the districts. Without using malaria epidemic monitoring charts, it was difficult to detect increased malaria cases at an early stage that would indicate emerging outbreaks. During these weeks the number of cases who had mixed infection was more than double than cases that had mono species in Midre-Genet and Wotet-Ber kebeles but in Zemene-Hiwot kebele plasmodium falciparum was more than tripled than others and under-fives were more affected.

To prevent subsequent malaria outbreaks the following action points put as recommendations:

- Improving the completeness/quality of data and use malaria monitoring chart

- Timely spray of IRS based on the transmission seasons so as to prevent outbreaks before malaria flare up.

- Health extension workers should work on environmental management by mobilizing the community
- LLINs should be replaced timely for those utilize appropriately

- Keep weekly malaria data and used it for early detection of malaria outbreaks

- Districts should allocate operational budget for spray

- Irrigation sites should be handled with care to prevent malaria outbreaks.

\section{References}

[1] World Health Organization. Malaria elimination a field manual for low and moderate endemic countries. Geneva: World Health Organization, 2004.

[2] Federal democratic republic of Ethiopia. National malaria guideline: 3rd edition; January 2012.

[3] Amhara regional health bureau. Annual report. Bahir Dar; 2011/12.

[4] Yolanda, Barbera, Lainez. An assessment of the intersectoral response to the malaria epidemic in Ethiopia;2003

[5] Technical guidelines for integrated disease surveillance and response in the African region. 2nd edition.

[6] Joseph Z. Losos. Routine and sentinel surveillance methods: Eastern Mediterranean health journal, volume two; issue 1, 1996, pages 46-50.

[7] Centers for Disease control and Prevention. Updated Guidelines for Evaluating Public Health Surveillance Systems: Recommendations from the Guidelines Working Group. MMWR 2001/50(RR13);1-35

[8] Ministry of Health of Ethiopia. National Malaria Indicator Survey. Addis Ababa; 2007.

[9] Ecology of Health and Diseases in Ethiopia. Addis Ababa: Shama Books:2006:565-75

[10] World Health Organization and UNICEF. Switzerland: World malaria report; 2010.

[11] Communicable diseases epidemiological profile (2007), Horn of Africa; pp.11-12. 